\title{
Elucidation of Genetic Markers for the Essential Oils Quantity in Mentha Plants F-AFLP \\ A.M.Adham ${ }^{1}$, M.Magdy ${ }^{2}$, R.M.Mostafa ${ }^{1}$, Y.G.Fiteha ${ }^{2}$, H.T.Elhifnawy ${ }^{2}$, M.A.Rashed ${ }^{2}$ and S.H.Abdel-Aziz $^{1}$ \\ ${ }^{1}$ Botany Dept, Faculty of Science, Benha Univ, Benha, Egypt \\ ${ }^{2}$ Genetics Dept, Faculty of Agriculture, Ain Shams Univ,Cairo, Egypt \\ E-Mail: Alyaa.alyaa@yahoo.com
}

Abstract

Medicinal plants are an essential source of therapeutic ingredients, being also the basis of traditional or original healing systems. Many medicinal plants belong to the family Lamiaceae. The Mint is the common name of about 25 perennial species of the genus Mentha. The essential oils of the Mentha species and their extracts are well documented and possess antimicrobial, antifungal, antiviral, pesticide, and antioxidant properties. The current study aimed to collect and genotype mint species from different sources using fluorescently labeled AFLP technique to asses the genetic variability and elucidate highresolution genetic markers related to oil content and/or oil quantity. A total of 11 mint samples were analyzed using AFLP technique; among the three primer pair combinations showed number of peaks ranged between 49 to 117 , with a minimum band size in base pair of $50 \mathrm{bp}$ to $661 \mathrm{bp}$. The overall mean of number of bands per individual is 99 , while the total number of polymorphic loci is 254 . Based on the maximum likelihood hybrid index method, two samples were found misclassified or possibly hybrids. Out of the 254 polymorphic bands, 16 were found specific to the high oil-content group [A] compared to 95 bands that were specific for the low oil-content group [B]. In group A, two bands, P1.23 and P2.28, were found fixed as present in group A while absent from group B, the bands were scored from the primer pairs 1 and 2 of 297 and 140 bp, respectively; and considered as positive markers to high-oil content. However, no negative marker was found. When the two possible hybrids were inspected, the positive marker number 1 [PP1.23] was found consistent and absents in the two samples, which increase its validity as a positive marker for high-oil content, while was not the same case for the second marker. Comparing both the whole dataset and the selected markers using AMOVA and PCoA, the elucidated markers should higher genetic differentiation among the studied groups than the whole dataset. The selected markers can be applied for markerassistant selection breeding program and early preselection of high-oil content samples for future studies related to the oil content in Mentha sp.

Keywords: AFLP, Essential Oils, Mentha sp, Minta, Molecular Markers .

\section{Introduction}

Plants are rich in phytochemical compounds that provide a source of food ingredients used for the treatment of different issues and ailments [1]. Medicinal plants are an important source of therapeutic ingredients, being also the basis of traditional or original healing systems, still widely used by the majority of populations in many countries [23] and referred to as traditional or ethnic medicine. Traditional medicine provides an outstanding health care service and can be used as alternate therapy. Many medicinal plants belong to the family Lamiaceae [11],[14] which is widely distributed in Europe, Asia, Africa, Australia, and North America [20], and inhabit multiple and diverse environments [9].

The Mint is the common name of about 25 perennial species of the genus Mentha [3]. Mentha species, one of the world's oldest and the most popular herbs, not only used as in food as flavor, spice, or perfumery and cosmetics, but with even higher medicinal value [19]. The mainly used as a therapy for flatulence, nausea, indigestion, anorexia, vomiting, and ulcerative colitis. Also, the essential oils of the Mentha species and their extracts are well documented and possess antimicrobial, antifungal, antiviral, pesticide, and antioxidant properties. Many studies show the bioactivity and health effects of spearmint the mint market beyond billions of dollars over the world [22]. Recent data, based on morphological, cytological, and genetic characteristics, have shown that genus Mentha can be classified into 42 species, 15 hybrids, and hundreds of subspecies, varieties, and cultivars [9].
The amplified fragment length polymorphism [AFLP] technique is a highly effective DNA Fingerprint technique for DNA, with different sizes and base composition, of any source or complexity. Also, this method demonstrates high discriminatory strength and good reproducibility potential allowing it to be useful in distinguishing both species and cultivars levels [24]. It has been found to be a rapid and reliable technique preventing obstacles from previously used markers such as RFLPs and RAPDs [2]. AFLP alleles can be fluorescently labeled allowing a parallel characterization of several samples in automatic genome analyzers. It is possible to obtain accurate information rapidly to allow for efficient identification and differentiation of species, subspecies and even genotypes [8].

The current study aimed to collect the genotype mint species from different sources using fluorescently labeled AFLP technique. In order to fingerprint the collected plants and to asses the genetic variability among them, while in parallel using genome scan approach to elucidate high-resolution genetic markers related to oil content and/or oil quantity[6].

\section{Material and methods 2.1 Material}

A total of 11 plants were collected from two different sources, ten samples [S01 to S10] from the Medicinal, Aromatic and Poisonous Plant Experiments Station, Faculty of Pharmacy, Cairo University, and one sample [S11] from El Zohour Showroom, Giza. For each plant the 
amount of essential oil was recorded according to each source description. The eleven plants can be distinguished into two major groups according to their essential oil amount, group A, of high amount of essential oil, which included plants no. $1-5$; and group B, of a low amount of essential oil, which included the plants no. $6-11$. Three fresh young leaves were collected from each plant in liquid nitrogen and transferred to the Molecular Biology laboratory, Faculty of Agriculture, Ain Shams University for DNA extraction, and AFLP analysis.

\subsection{Methods}

\subsubsection{DNA extraction protocol from mint oily leaves}

Before DNA extraction, all material was rinsed in $25 \%$ Chloroform for 2 min to reduce oil content and then rinsed with $\mathrm{ddH}_{2} \mathrm{O}$ for $5 \mathrm{~min}$. The washed material was transferred into sterile mortars and ground by pestle after chilling in liquid nitrogen. Grinded material was transferred to $2 \mathrm{ml}$ tubes and $400 \mathrm{ul} 2 \%$ CTAB extraction buffer supplemented with 1\% BME and 4\% PVP was applied. The homogenized tissue was transferred to a $60^{\circ} \mathrm{C}$ bath for 30 minutes, followed by undercooling centrifugation at $15,000 \mathrm{rpm}$ for $5 \mathrm{~min}$. The supernatant was washed by chloroform/isoamyl alcohol [24:1] for 5 seconds then centrifuged at 15,000 rpm for $1 \mathrm{~min}$ twice. The final upper aqueous phase was transferred to fresh tube, and 0.5 equal volume of isopropanol was added and incubated at $-20^{\circ} \mathrm{C}$ for 60 minutes. Afterward, samples were subject to centrifugation at $15,000 \mathrm{rpm}$ for $10 \mathrm{~min}$. The resulted pellet was washed with ice-cold $70 \%$ ethanol and dried out at room temperature. Washed pellet was

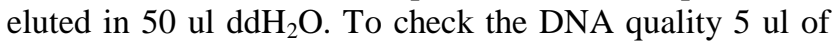
each sample was visually tested by $0.5 \%$ [1g/100ml] agarose gel electrophoresis. DNA appears as sharp bands when viewed using the UV transilluminator. Successful DNA extracts were stored at $-20{ }^{\circ} \mathrm{C}$.

\subsubsection{AFLP protocol}

The original protocol of [1] was used with a single modification in the labeling type, as primers were labeled by fluorescent instead of radioactive material. Adaptor and primer sets were all synthesized by Eurofins [Germany], as shown in Table (1) and prepared to the final concentrations accordingly. The protocol consists of three main steps, a] Digestion by restriction enzymes and ligation of adaptors [Dig-Lig], b] Pre-selective PCR and c] Selective PCR. All three steps were prepared and applied according to the original protocol and tested for success using $1 \%$ [1g/100ml] agarose gel electrophoresis and visualized under UV transilluminator. Selective PCR products were shipped in dry ice for fragment analysis at Secugen Inc. [Madrid, Spain].

\subsection{Data analysis}

Chromatograms were analyzed using PeakScanner 2 [Applied Biosystems, USA], while band scoring and pseudo-gel imaging were performed using RawGeno package on R. FAMD software [7] was used to filter rare alleles $[0.5 \%>$ alleles presence per individual $<95 \%$ ], and to estimate loci polymorphism, group reallocation, genetic dissimilarity matrix and principal coordinate analysis [PCoA].

\section{Results}

Quality Check, Sample Filtering, Scoring and Polymorphism

The AFLP analysis of the 11 mint samples included chromatograms quality check, filtering, and scoring. Among the three primer pair combinations showed number of peaks ranged between 49 to 117 , with a minimum band size in base pair of $50 \mathrm{bp}$ to $661 \mathrm{bp}$. The maximum number of bands was 135 and scored from primer pair 1 [ACA/CTC] while the lowest was 40 scored from primer pair 2 [AGG/CTC], while the remained primer pair [ATA/CTC] scored 79 bands. The overall mean of number of bands per individual is 99 , while the total number of polymorphic loci is 254 [100\% polymorphism; Table (2), Fig (1).

Table (1) Primers and Adaptors [P/A] name and sequence. The fluorescent label is written in bold

\begin{tabular}{lccc}
\hline P/A & 5'-Sequence -'3 & P/A & 5'-Sequence -'3 \\
\hline EcoR1-A1 & CTCGTAGACTGCGTAC & Mse+ C & GATGAGTCCTGAGTAAC \\
\hline EcoR1-A2 & AATTGGTACGCAGTC & Eco +ACA & FAM-GACTGCGTACCAATTCACA \\
\hline Mse1-A1 & GACGATGAGTCCTGAG & Eco +AGG & HEX-GACTGCGTACCAATTCAGG \\
\hline Mse1-A2 & TACTCAGGACTCAT & Eco +ATA & CY3-GACTGCGTACCAATTCATA \\
\hline Eco + A & GACTGCGTACCAATTCA & Mse + CTC & GATGAGTCCTGAGTAACTC \\
\hline
\end{tabular}

Table (2) AFLP scoring statistics and polymorphism for three primer pair combinations

\begin{tabular}{lccccccccc}
\hline Primer pair & Dye color & $\begin{array}{c}\text { Samples } \\
\text { Kept }\end{array}$ & $\begin{array}{c}\text { Min } \\
\text { peaks }\end{array}$ & $\begin{array}{c}\text { Max } \\
\text { peaks }\end{array}$ & $\begin{array}{c}\text { Min size } \\
{[\text { bp] }}\end{array}$ & $\begin{array}{c}\text { Max } \\
\text { size [bp] }\end{array}$ & MNP* & $\begin{array}{c}\text { Polymorphic } \\
\text { Bands }\end{array}$ & $\%$ P** \\
\hline ACA/CTC & Blue & 11 & 49 & 96 & 50 & 574 & 50 & 135 & $100 \%$ \\
\hline AGG/CTC & Green & 11 & 32 & 71 & 103 & 302 & 13 & 40 & $100 \%$ \\
\hline ATA/CTC & Yellow & 11 & 99 & 117 & 50 & 661 & 34 & 79 & $100 \%$ \\
\hline Total & - & 11 & 180 & 284 & 50 & 661 & 99 & 254 & $100 \%$ \\
\hline
\end{tabular}

* MNP: Mean number of bands per individual; ** \%P: Percentage of polymorphism. 
Table (3)The maximum likelihood hybrid index estimated for each sample

\begin{tabular}{lccccccccccc}
\hline log-likelihood & S01 & S02 & S03 & S04 & S05 & S06 & S07 & S08 & S09 & S10 & S11 \\
\hline Group A & -54 & -41 & -48 & -44 & -55 & -86 & -72 & -59 & -89 & -93 & -83 \\
\hline Group B & -68 & -61 & -69 & -61 & -62 & -81 & -73 & -66 & -77 & -88 & -80 \\
\hline Difference & 14 & 21 & 22 & 18 & 7 & 5 & 1 & 7 & 12 & 4 & 3 \\
\hline Group H0 & A & A & A & A & A & B & B & B & B & B & B \\
\hline Allocation & A & A & A & A & A & B & None & A & B & B & B \\
\hline
\end{tabular}

\section{Group reallocation and samples sorting}

Applying the maximum likelihood hybrid index method, the group reallocation of samples was performed based on all alleles. Using the minimum log-likelihood value criteria, samples no. 7 was defined as outlier, while sample no. 8 was reallocated from group [B] to group [A], and alternatively, they may be defined as possible hybrid. Based on the new reallocation, the samples were regrouped accordingly and retained for further analysis Table (3).

Table (4) Binary data of the two newly elucidated molecular markers for high oil content showed for all samples

\begin{tabular}{|c|c|c|c|c|c|c|c|c|c|c|c|c|}
\hline \multirow{2}{*}{$\begin{array}{l}\text { Locus } \\
\text { code }\end{array}$} & \multirow{2}{*}{$\begin{array}{c}\text { Molecular } \\
\text { weight }\end{array}$} & \multicolumn{5}{|c|}{ Group A } & \multicolumn{4}{|c|}{ Group B } & \multicolumn{2}{|c|}{ Hybrids } \\
\hline & & S01 & S02 & S03 & S04 & S05 & S06 & S09 & S10 & S11 & S07 & S08 \\
\hline PP1.23 & $297 \mathrm{bp}$ & 1 & 1 & 1 & 1 & 1 & 0 & 0 & 0 & 0 & 0 & 0 \\
\hline PP2.28 & $140 \mathrm{bp}$ & 1 & 1 & 1 & 1 & 1 & 0 & 0 & 0 & 0 & 1 & 0 \\
\hline
\end{tabular}

\section{Genetic differentiation and marker validation}

Based on the acquired information, the AMOVA analysis was performed between the two groups and within each group. The case where the grouping was based on the sampling source information, the percentage of variation estimated among the two groups was $18 \%$ compared to the variation within each group of $82 \%$, reflecting high genetic differentiation within each group. However, when the regrouping based on the current analysis was set, the variation within each group decreased to $61 \%$, and the genetic differentiation between the two groups increased to 39 compared to the default grouping Fig (2), which indicate a better homogenization among the samples of the same group was achieved.
The specific bands were used to calculate the dissimilarity and PCoA graph in order to validate the sample grouping based on the whole dataset and the selected markers. The PCoA based on all dataset showed a more considerable variation than the selected [elucidated markers], where the samples were scattered in the all dataset case, and S08 were very proximate to group A. In contrast, the PCoA based only on the elucidated markers, the two groups were clearly defined, and the hybrid was potentially detected, which reflect the power of the elucidated marker to detect the homogenate lines and the possible hybrid in relation to the oil content.
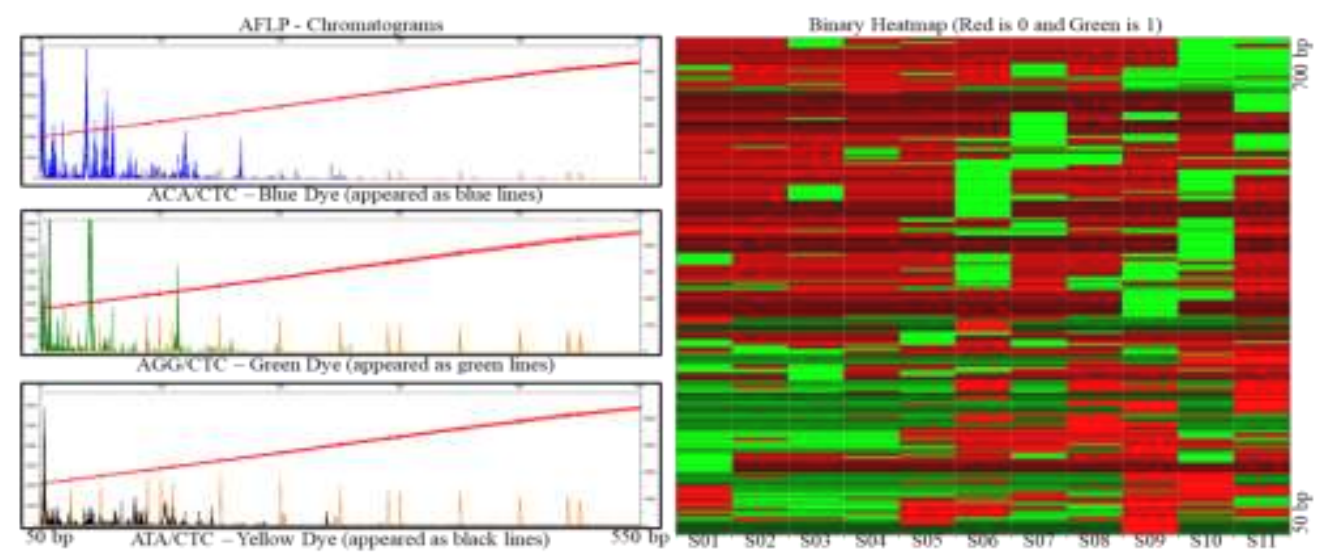

Fig (1) AFLP chromatograms [left] and the scored bands [right]. Chromatograms of the three primer pairs are shown by their dye color over all the 11 samples, blue [FAM], green [HEX], and yellow [CY3]; orange peaks are the GS500LIZ standard size = DNA marker, ranging from $50 \mathrm{bp}$ to $550 \mathrm{bp}$. The heatmap for the scored bands is shown contrasting two colors, red for absence bands, and green for present. 


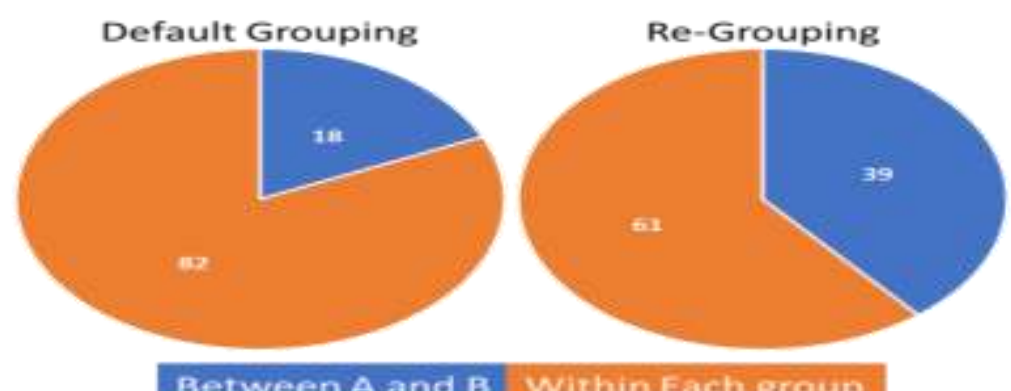

Fig (2) Pie chart of AMOVA results based on the AFLP data, calculated twice, one comparing the samples in two groups based on the sampling source information [default grouping; left] and two, comparing the samples un two groups based on the information acquired by the current results [Re-grouping; right].
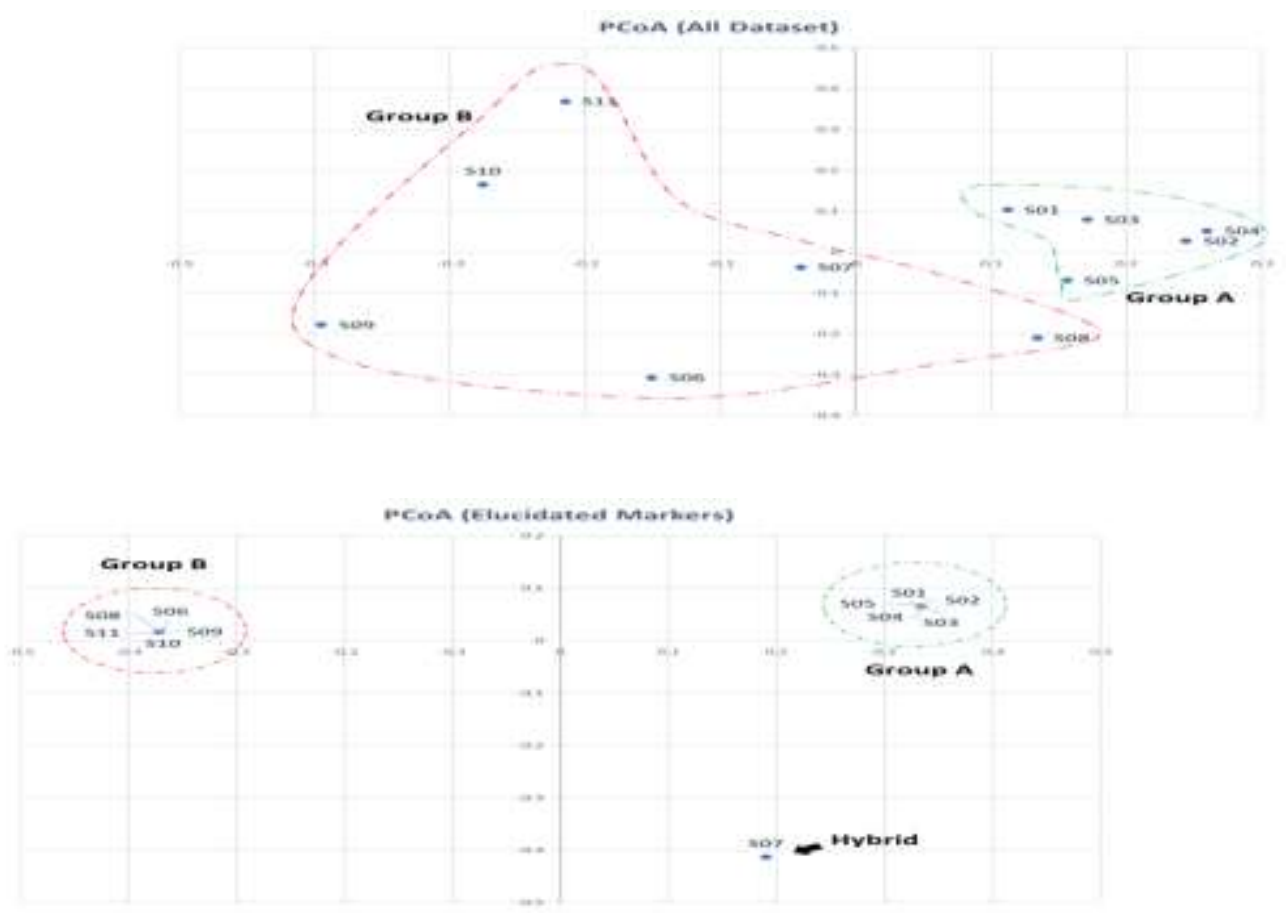

Fig (3) PCoA of the AFLP scored bands as the all bands [Above] and the selected markers [below]. The two groups are defined by colored shaps [above] and circles [belwoe], in addition to the hybrid sample S07 [indicated by black arrow, below].

\section{Specific alleles for oil content-based groups}

Based on the grouping according to the sampling source information; out of the 254 polymorphic bands, 16 were found specific for the high oil-content group [A] compared to 95 bands that were specific for the low oilcontent group [B]. After regrouping based on the AFLP maximum likelihood hybrid index and structure results, out of the 254 polymorphic bands, 26 were found specific for group [A] compared to 81 bands that were specific for group [B].

After removing the conflicting samples [S07 and S08], and by using visual inspection in Excel, the private bands were filtered to fixed bands within each group. In group A, two bands, P1.23 and P2.28, were found fixed as present in group $\mathrm{A}$ while absents from group $\mathrm{B}$, the bands were scored from the primer pairs 1 and 2 of 297 and $140 \mathrm{bp}$, respectively; and considered as positive markers to highoil content. However, no negative marker was found. When the two possible hybrids [S07 and S08] were inspected, the positive marker number 1 [PP1.23] was found consistent and absents in the two samples, which increase its validity as a positive marker for high-oil content, while was not the same case for the second marker, as its hybrid potential had its influence on the appearance of a band in S07.

\section{Discussion}

The use of molecular markers to evaluate the genetic diversity and relationships among individuals and populations is beneficial as many polymorphic loci can be procured in a relatively short time and at low cost, without any prior knowledge of the genome of the species under 
study [4]. AFLP has emerged as a powerful technique for cultivar identification and fingerprinting [18]. The present results are relevant and comprehensive work to assess the genetic variability and detect multiple polymorphisms among mint species. Furthermore, using genome scan approach to elucidate high-resolution genetic markers related to oil content and/or oil quantity.

The results of the AFLP validation study showed different banding patterns for each group/accession. An analysis of AFLP data from three primer pair combinations assayed in this study revealed important differences in genetic variation between and within different samples of Mentha. The total number of polymorphic loci is 254 [100\% polymorphism]. This corresponds to extremely high polymorphic rates when compared to other studies where AFLP markers were used; [10] discovered that a total of the genetic variation between [15] L. was about $33.7 \%$, and the variation resided within accessions was $66.3 \%$. [12] assessed the genetic diversity of 20 Iranian populations of Bunium persicum and reveled to 228 [75\%] polymorphic bands. [5] assessed the genetic diversity to evaluate the relationships within Mentha, representing five species and three hybrids from different geographical origins and found the polymorphic proportion of markers for all accessions studied ranged from $50 \%$ to $60 \%$ but using a less reproducible marker system [ISSR]. The AFLP marker allowed the reallocation of samples among the two studied groups and was successfully capable of define the hybrids and the misclassified samples [16].

The AMOVA results indicating high differentiation value within the Mentha populations [Fst $=82 \%$ ]. Several studies used the AMOVA analysis to display the significant differences in genetic variation between and within the populations and individuals. Similarly, [21] determined a variation within Spathoglottis plicata populations from different locations in Peninsular Malaysia. The Fst value equal to $78.5 \%$, indicating a potential restricted gene flow [17].

On the bases of molecular marker validation, the AMOVA was found useful to compare the whole dataset and the selected markers, indicating higher differentiation value for the later, which indicates the usefulness of the selected marker for the differentiation of the samples based on their oil-content group. The selected markers can be applied for marker-assistant selection breeding program and early preselection of high-oil content samples while avoiding mis-classified ones, for future studies related to the oil content in Mentha sp [24].

\section{References}

[1] p.Vos,Hogers, M.Bleeker, T. V.d ,AFLP:a new teachnique for DNA fingerprinting .Nucleic acids Research, Vol.23, PP.4407-4414,1995.

[2] R.T.Folkertsma, R.V.Voort, J.N.A, De Groot,Gene pool similarities of potato cyst nematode populations assessed by AFLP analysis. MPMI-Molecular Plant Microbe Interactions, Vo.9[(1), PP. 47-54,1996.

[3] B.M.Lange,R.Croteau,Genetic engineering of essential oil production in mint. Current Opinion in Plant
Biology,Vol. 2(2), PP.139-144,2003.

[4] H.Nybom, I.V.Bartish,Effects of life history traits and sampling strategies on genetic diversity estimates obtained with RAPD markers in plants. Perspectives in Plant Ecology, Evolution and Systematics, Vol. 3(2), PP.93-114,2000.

[5] V.Gobert, S.Moja, M.Colson and P.Taberlet , Hybridization in the section Mentha [Lamiaceae] inferred from AFLP markers. American J., of Botany, 89[12], 2017-2023. https://doi.org/.Vol..10.3732/PP.89.12,2002.

[6] H.Nybom,Comparison of different nuclear DNA markers for estimating intraspecific genetic diversity in plants. Molecular Ecology, Vol . 13(5), PP.1143$1155,2004$.

[7] P.M.Schluter, S.A.Harris,Analysis of multilocus fingerprinting date sets containing missing date . Molecular Ecology Notes 6:569-572,2006.

[8] B.M,Lawrence,Mint: the genus Mentha. CRC Press: Boca Raton, FL, USA,2006.

[9] M.Lawrence: Mentha: Economic uses. In Mint: The Genus Mentha. CRC Press: Boca Raton, FL, USA, 519-522,2007.

[10] M.A,El-Esawi , Genomic characterization and genetic improvement of some Lactuca sPP. Tanta University, Egypt,2008.

[11] A.Kahraman, F.Celep,Dogan, M, Morphology, anatomy and palynology of Salvia indica L.[Labiatae]. World Applied Sciences J.,, 6 vol.2, PP.289296,2009.

[12] M.Pezhmanmehr, M.E.Hassani, F.Jahansooz, A.Najafi , Assessment of genetic diversity in some Iranian populations of Bunium persicum using RAPD and AFLP markers. Iranian J., of Biotechnology, vo.7(2), PP.93-100,2009.

[13] S.R.Weerakoon, O.J.Weerasena, P.K.DPeiris, S.Somaratne, Assessment of genetic variation within mustard [Brassica juncea [L.] Czern \& Coss] germplasm in Sri Lanka using fluorescent-based amplified fragment length polymorphic DNA markers. International J., of Biotechnology \& Biochemistry, vol .6(5),PP. 757-769,2010.

[14] A.N.Chaker, H.Boukhebti, F.Sahli, R.Haichour, Morphological and anatomical study of two medicinal plants from genus mentha. Advances in Environmental Biology,vol: 5(2]), PP.219-221,2011.

[15] S.Christensen, R.Bothmer, G.Poulsen, L.Maggioni : AFLP analysis of genetic diversity in leafy kale [Brassica oleracea L. convar. acephala [DC.] Alef.] landraces, cultivars and wild populations in Europe. Genetic Resources and Crop Evolution, 58(5), 657$666,2011$.

[16] Z.Faltusova, L.Kuera, J.Ovesna: Genetic diversity of Brassica oleracea var. capitata gene bank accessions assessed by AFLP. Electronic J., of Biotechnology,Vol. 14(3), PP. 11,2011.

[17] M.Erome, S.Mace, X.Dousset, B.Pot :Genetic diversity analysis of isolates belonging to the Photobacterium phosphoreum species group collected from salmon products using AFLP fingerprinting. 
International J., of Food Microbiology,vol: 217, PP.101-109,2016.

[18] M.El-Esawi, A.K.Germaine, P.Bourke and R.Malone: AFLP analysis of genetic diversity and phylogenetic relationships of Brassica oleracea in Ireland. Comptes Rendus - Biologies, 339[5-6], 163-170. https://doi.org/Vol.10.1016/j.PP. 03.002,2016.

[19] F.Brahmi , M.Khodir, C.Mohamed, and D.Pierre: Chemical Composition and Biological Activities of Mentha Species. Aromatic and Medicinal Plants Back to Nature, 47-80. https://doi.org/Vol.10:PP.5772/67291,2017.

[20] N.Mamadalieva, D.Akramov, E.Ovidi, A.Tiezzi: Aromatic medicinal plants of the Lamiaceae family from Uzbekistan: Ethnopharmacology, essential oils composition, and biological activities. Medicines, Vol. 4([1),PP. 8,2017.

[21] C.F.Ginibun, P.Arens, B.Vosman, S.Bhassu : Genetic diversity of endangered terrestrial orchids' Spathoglottis plicata'in Peninsular Malaysia based on AFLP markers. Plant Omics, Vol. 11(3),PP.135,2018.

[22] A.K.Leong, A.B.Shori, S.A.Baba: Bioactivity and health effects of Mentha spicata. Integrative Food, Nutrition and Metabolism, 5(1), 1-2. https://doi.org/Vol.:10.15761/PP.1000203,2018.

[23] J.Sharifi-Rad, G.S.Tayeboon, F.Niknam, M.SharifiRad: Veronica persica Poir. extract-antibacterial, antifungal and scolicidal activities, and inhibitory potential on acetylcholinesterase, tyrosinase, lipoxygenase and xanthine oxidase. Cellular and Molecular Biology, Vol.46(8), PP. 50-56,2018.

[24] G.Bertani, M.Sardaro L.S, E.Neviani, and C.Lazzi:AFLP protocol comparison for microbial diversity fingerprinting. J., of Applied Genetics,vol:60[2],PP.217-223. https://doi.org/10.1007 /s13353-019-00492-0,2019. 